\title{
Sustainable technologies for waste reduction and pollutants removals
}

\author{
Pau Loke Show ${ }^{1}$ - Suchithra Thangalazhy-Gopakumar ${ }^{1}$ - Dominic C. Y. Foo ${ }^{1}$
}

Published online: 14 January 2021

(c) Springer-Verlag GmbH Germany, part of Springer Nature 2021

Continual growth in industrial activities and in transportation and residential sectors of the economy has led to steadily higher quantities of miscellaneous wastes and pollutants released into the surroundings environment. This special issue is offering insights on technological advancements and prospects, which are centred on three key topics: (1) wastewater treatment, management and recycling, (2) microbiome-mediated pollutants reduction and remediation and (3) solid wastes and agricultural residues treatment, management and recycling. A total of fourteen papers from outstanding researchers from several countries were accepted for publication after thorough peer reviews.

Microalgae cultivation with wastewater has attracted global attention as a means to remove harmful residual nutrients and produce low-cost biomass for biorefineries. Also, assimilation of nitrogen and phosphorous in microalgae can be recycled via production of biofertilisers. The resulting biomass can also be used to produce bioenergy, food, animal feed and biopharmaceutical, while the generated oxygenated effluent can be discharged into the water bodies. In addition, cultivating microalgae in the domestic wastewater is an alternative approach to conventional activated sludge processes for simultaneous pollutants removal and biomaterials production for biorefinery.

Novel bacterial strains were tested for decolourisation and degradation of sulphonated azo dyes. Azo dyes are suspected to be toxic to human health and the environment. These dyes are typically used in paper, textiles and cosmetics industries. Another frequently used consumer product is motor

Pau Loke Show

PauLoke.Show@nottingham.edu.my;

showpauloke@gmail.com

Suchithra Thangalazhy-Gopakumar

Suchithra.Thangalazhy@ nottingham.edu.my

Dominic C. Y. Foo

Dominic.Foo@nottingham.edu.my

1 Department of Chemical and Environmental Engineering, Centre for Green Technologies, University of Nottingham Malaysia, Broga Road, 43500 Semenyih, Malaysia oil, which may be harmful if released because of its contents of heavy metals, polychlorinated biphenyls and polycyclic aromatic hydrocarbons. The hazard comes from absence of an appropriate treatment, such as wilful dumping to land and waterways including sewers. Used lubricating oils are considered valuable resources. The recycling of these oils by solvent extraction using a ternary solvent consisting of 2-propanol, 1-butanol and methyl ethyl ketone followed by a vacuum distillation unit has been reported in one of the papers.

The fabrication of superhydrophobic/superoleophilic materials with water contact angle $>150^{\circ}$ and oil contact angle $<5^{\circ}$ for oil spill clean-up has attracted considerable research interest owing to these materials' high adsorption selectivity toward various oils. Herein, a distinctive ecofriendly corn straw material with preferable superhydrophobicity and superoleophilicity for separating oil from liquid mixtures was studied. The modified corn straw provided an eco-friendly alternative for the clean-up of oil spills, as well as a means to relieve the environmental problem of agricultural waste disposal. Also, there was a study on the sustainable cultivation via waste soybean extract for higher vaccenic acid production by purple non-sulphur bacteria. Furthermore, researchers employed Protamex protease (endopeptidase) to hydrolyse tuna's black muscle as to produce fish protein hydrolysate (i.e. a nutritional food powder). This study has provided an efficient procedure to solve the solid waste from the seafood manufacturing factories while manufacturing a nutritional food product. Moreover, a detailed assessment of the yield and quality of bio-oil produced from the fast pyrolysis process of date seeds and date syrup waste was reported. The pyrolytic oil of date seeds and syrup waste could be further refined and employed as building blocks for several value-added products in biofuel production and pharmaceutical/flavouring agents.

Ethylene is a basic raw material of petrochemical industry. An optimisation method for analysing the reaction systems of the ethane thermal cracking furnace, predicting its performance and identifying the optimal parameters was studied. Besides, a novel catalytic technique, i.e. glycerol 
esterification with oleic acid as catalyst, was experimented to produce mono- and dioleate (GMO and GDO) from bioglycerol (a renewable resource and by-product of biodiesel). The advantage of this method is the direct production of GMO and GDO through the catalytic approach compared to the conventional method that requires transesterification and distillation processes. Nowadays, green synthesis of nano-titania has gained substantial attention as an effective, safe and environmentally friendly practice. By doing so, the surface area is increased by reducing the agglomeration of nanoparticles and shifting the absorption edge to the visible range. Limitations of poor surface properties and meagre visible light absorption capacity of titania stand as barriers to its use as an effective visible light-active photocatalyst. To conclude, this special issue has reported research on multidisciplinary areas, and we strongly believe these are beneficial in achieving a positive impact on the environment.

Publisher's Note Springer Nature remains neutral with regard to jurisdictional claims in published maps and institutional affiliations. 